\title{
Verletzung der Quadrizeps- und Patellarsehne
}

\author{
Mirco Herbort, Christoph Domnick, Michael J. Raschke
}

\section{Zusammenfassung}

Strecksehnenrupturen des Kniegelenks sind seltene, aber relevante Verletzungen, die zu einer ausgeprägten Einschränkung der Funktion des Streckapparats und somit der gesamten unteren Extremität führen können. Ursache für diese Rupturen sind i.d.R. degenerative Vorschäden der Quadrizepsoder Patellarsehne, die im Falle einer maximalen Anspannung der Quadrizepsmuskulatur im Rahmen eines inadäquaten Traumas zu einer Ruptur der Sehne führen können. Die Diagnosestellung ist im Falle einer vollständigen Ruptur meist im Rahmen einer gründlichen klinischen Untersuchung möglich. Bei unklarem klinischem Befund im Falle von Teilrupturen oder Rerupturen sollte eine Sonografie und/ oder eine MRT-Diagnostik erfolgen. Die Therapie erfolgt i.d.R. operativ mittels direkter Adaptation der Sehnenenden mit Naht mittels U-, Kessler- oder Bunnell-Nähten. Die Fixation mit den knöchernen Ansatzstellen im Bereich der Patella oder Tuberositas kann entweder mittels transossärer Nähte oder Knochenankern erfolgen. Bei chronischen Rupturen oder Rerupturen ist meist eine Sehnenaugmentation mit autologem Sehnenmaterial notwendig. Die Nachbehandlung sollte unter Schonung der Nähte, jedoch mit frühen passiven Übungen der Beweglichkeit des Kniegelenks erfolgen, um Komplikationen zu verhindern.

\section{Injury of the Quadriceps and Patellar Tendon}

Extensor tendon ruptures of the knee joint are rare but relevant injuries, which may severely restrict the function of the extensor mechanism and the entire lower extremity. Such ruptures are usually caused by previous degenerative damage to the quadriceps or patellar tendon, which may lead to a tendon rupture in the event of maximum tension of the quadriceps muscle as part of an inadequate trauma. A complete rupture is usually diagnosed during a thorough clinical examination. If clinical findings are ambiguous or in the case of partial ruptures or re-ruptures, an ultrasound and/or an MRI scan should be performed. Treatment usually involves surgery with direct apposition of the tendon ends with $U$ sutures, Kessler or Bunnell sutures. Fixation with the bony insertions in the area of the patella or tibial tuberosity can be performed either by transosseous sutures or bone anchors. Chronic ruptures or re-ruptures usually require tendon augmentation with autologous tendon material. Postoperative rehabilitation should be performed with care to protect the sutures, but should include early passive range of motion exercises of the knee joint in order to prevent complications.

\section{Quadrizepssehnenruptur}

\section{Anatomie}

Die Quadrizeps- und Patellarsehne dienen im Kniegelenk zur Kraftübertragung der Streckmuskulatur. Diese besteht aus dem 4-köpfigen $M$. quadriceps, welcher entsprechend in 4 Anteile aufgeteilt werden kann (M. rectus femoris, M. vastus lateralis, M. vastus medialis und M. intermedius). Der M. rectus femoris stellt den oberflächlichen Anteil des M. quadriceps dar. Er verläuft mit einem Teil seiner Fasern über die Oberfläche der Patella hinweg in die Patellarsehne hinein und bildet so den Reservestreck-

OP-JOURNAL 2015; 31: 174-180

(c) Georg Thieme Verlag KG Stuttgart · New York DOI http://dx.doi.org/10.1055/s-0041-110845 apparat. Der Vastus medialis bildet den medialen Muskelanteil der Quadrizepsmuskulatur. Er kann wiederum nach Berücksichtigung des Verlaufs und der Funktion der Muskelfasern in den M. vastus medialis longus und M. vastus medialis obliquus eingeteilt werden. Aufgrund der 4 unterschiedlichen Muskelanteile mit ihren typischen Faserverläufen kommt es in der gemeinsamen Insertionssehne (Quadrizepssehne) zu einem faserigen Aufbau mit 2-4 unterschiedlichen Lagen, welche durch Fettschichten von einander separiert werden [31].

Wegen der typischen Druckbelastung des Sehnengewebes unter Flexionsstellung des Kniegelenks befindet sich im Abstand von $10 \mathrm{~mm}$ proximal der Patella im posterioren Blatt der Quadrizepssehne ein gefäßarmes Areal [27]. Aufgrund der geringen Gefäßversorgung weist dieses Areal besonders häufig degenerative Veränderungen auf $[27,35]$. Da es sich bei Quadrizepssehnenrupturen i.d.R. um degenerative Risse handelt, sind die Rupturstellen häufig in diesem gefährdeten Bereich zu finden.

\section{Epidemiologie/Ätiologie}

Quadrizepssehnenrupturen können in seltenen Fällen durch Hochrasanztraumata des Kniegelenks verursacht werden. In den meisten Fällen handelt es sich bei dieser Ruptur jedoch um eine Folge eines inadäquaten Traumas auf Grundlage einer degenerativen Vorschädigung des Sehnengewebes. Als typischer Traumamechanismus wird eine maximale Anspannung der Quadrizepsmuskulatur gegen Widerstand angegeben. In der aktuellen Literatur gibt es 
keine genauen Zahlen über die Häufigkeit der Ruptur der Quadrizeps- oder Patellarsehne. Es sind jedoch Häufigkeitsverteilungen im Vergleich $\mathrm{zu}$ anderen Sehnenrupturen publiziert worden. So kommt eine Achillessehnenruptur 16mal häufiger vor als eine Patellarsehnenruptur [18]. Mit einem Verhältnis von $6: 1$ ist das männliche Geschlecht häufiger von einer Quadrizepssehnenruptur betroffen [12].

\section{Pathomechanismus}

Voraussetzung für das Erleiden einer Quadrizepssehnenruptur ist meistens der degenerative Vorschaden der Sehne. Typische Vorschädigungen, die im Falle einer Quadrizepssehnenruptur gefunden werden, sind Verfettung, hypoxische Tendinopathie, idiopathische Tendinopathie, kalzifizierende Tendinopathie und multiple degenerative Veränderung $[12,14,15]$.

Als Ursache für diese degenerativen Veränderungen der Quadrizepssehne werden die folgenden Erkrankungen und Faktoren diskutiert $[8,12,14,15,26]$ :

- langzeitliche systemische oder lokale Therapie mit Glukokortikoiden

- endokrine Erkrankungen (Hyperurikämie, Diabetes mellitus 1 bzw. 2, Adipositas, Niereninsuffizienz, Hyperparathyreoidismus, metabolisches Syndrom)

- Anabolikaabusus

- kompromittierende Gefäßversorgung der Sehne

- lokale Ischämie durch mechanische Belastung (z.B. hockende Tätigkeit)

\section{Diagnostik}

Die Diagnosestellung der kompletten Quadrizepssehnenruptur erfolgt i.d.R. bereits anhand des klinischen Befunds.

Neben der typischen suprapatellaren Delle, welche im Verlauf aufgrund des Hämatoms nicht mehr eindeutig palpierbar sein muss, spricht eine Unfähigkeit der aktiven Streckung des Kniegelenks gegen Widerstand für eine Quadrizepssehnenruptur.

Zur differenzialdiagnostischen Unterscheidung zur Patellarsehnenruptur sollte v.a. die Lokalisation von Einblutungen wie auch die Stellung der Patella in der klinischen Untersuchung beachtet werden. Neben einer dezidierten klinischen Untersuchung gehört eine genaue Anamneseerhebung zur adäquaten Behand- lung. Dabei sollten die folgenden Themenbereiche abgefragt werden: klinische Symptome, Schmerzanamnese, Verletzungsmechanismus, Medikamentenanamnese (Kortison, Anabolika), systemische Grunderkrankungen [12,26].

Das apparative Diagnostikum der Wahl einer Quadrizepssehnenruptur ist die Sonografie, mit deren Hilfe die klinische Verdachtsdiagnose schnell und kostengünstig eindeutig bestätigt werden kann. Insbesondere im Falle einer Teilruptur, welche bei der klinischen Untersuchung die Diagnosestellung erschweren kann, bietet die Sonografie mit Nachweis von Hämatom besonders in der Frühphase die schnelle Bestätigung des Verdachtsbefunds. Ein weiterer großer Vorteil der Sonografie ist die Möglichkeit der dynamischen Untersuchung, die eine allumfassende Analyse der Ruptur ermöglicht.

Neuere Untersuchungen konnten jedoch in einer retrospektiven Studie ernüchternde Ergebnisse bez. der Diagnosestellung von Total- und Teilrupturen des Kniestreckapparats verzeichnen [34]. In dieser Studie zeigte sich v.a. bei übergewichtigen Patienten eine deutliche Einschränkung der Genauigkeit der Diagnosestellung mittels Sonografie. In diesen Fällen sollte bei klinischem Verdacht auf eine Affektion des Streckapparats die Diagnostik mittels MRT erweitert werden.

Das MRT spielt ansonsten in der Primärdiagnostik eine eher untergeordnete Rolle. Im Falle einer Reruptur oder degenerativen Teilruptur bietet die MRT-Bildgebung jedoch wichtige Zusatzinformationen wie Status der Degeneration, Ausmaß der Dehiszenz mit qualitativem Status des Sehnengewebes. Diese Informationen sind im Falle einer Revision von größter Bedeutung und bestimmen das operative Vorgehen [22]. Weiterhin kann eine durchgeführte MRT-Diagnostik objektive und stichhaltige Informationen für eine gutachterliche Beurteilung bieten.

Mithilfe einer Röntgenaufnahme (insbesondere im seitlichen Strahlengang) können zum einen die Patellastellung bzw. -höhe, eine ventrale Abkippung, zum anderen auch mögliche knöcherne Avulsionen (insbesondere bei Kindern) und degenerative Veränderungen mit z.B. Kalzifikationen dargestellt werden.

\section{Therapie}

Teilruptur

Im seltenen Fall einer Teilruptur der Quadrizepssehne kann grundsätzlich ein konservatives Vorgehen empfohlen werden [22]. Dies gilt insbesondere bei erhöhtem peri- und postoperativem Risiko. Jedoch sollte gerade bei älteren Patienten eine langzeitige Immobilisation verhindert werden, welche sich bei konservativem Vorgehen erheblich verlängern kann.

\section{Totalruptur}

Im Falle einer vollständigen Ruptur der Quadrizepssehne ist die operative Intervention indiziert. Hierbei wird im eigenen Vorgehen die offene Naht der zerrissenen Sehne favorisiert.

Der Operationszeitpunkt sollte idealerweise innerhalb von 48-72 Stunden gewählt werden, um eine optimale Voraussetzung zur Heilung der Sehnenruptur zu ermöglichen [20,32].

Zur Vorbereitung der Operation sollten beide Kniegelenke steril abgewaschen und während der Operation frei zugänglich sein. Dieses Vorgehen erlaubt den intraoperativen Vergleich der Patellahöhe klinisch und evtl. radiologisch (seitl. Strahlengang). Standardzugang zur Naht der Quadrizepssehne ist die mediane Hautinzision. Diese erlaubt im Falle einer späteren Revisionssituation oder Folgeoperation (Knie-TEP etc.) sämtliche Möglichkeiten. Über diesen Zugang wird die Quadrizepssehnenruptur vollständig freigelegt, und degenerative und stark zerrissene Sehnenanteile werden débridiert. Eine histologische Probeentnahme und Aufarbeitung kann im Falle eines Berufsunfalls eine spätere Einstufung der Kausalitäten erleichtern und wird somit empfohlen. Anschließend sollte im Falle einer akuten Ruptur eine vollständige spannungsfreie Adaptation und Naht der zerrissenen Sehne angestrebt werden. Die genaue Nahttechnik sollte an die Höhe der Ruptur bzw. an den Abstand der Ruptur zur Patella und zum muskulotendinösen Übergang angepasst werden.

In der aktuellen Literatur wird jedoch grundsätzlich eine Durchflechtungsnaht in Kombination mit einer Rahmennaht empfohlen $[12,20,26]$. 
Die Fixation der Quadrizepssehne bzw. der Fäden, mit denen die Quadrizepssehne in Masson-Allen- oder Krackow-Technik angenäht worden ist, kann grundsätzlich auf zweierlei Art und Weise erfolgen:

- Entweder werden in Längsrichtung transossäre Bohrungen - z.B. mit einem 2,0-mm-Bohrer-durchgeführt, mit denen die Quadrizepssehne am proximalen Patellapol fixiert wird,

- oder die knöcherne Fixation der Sehne erfolgt über die Platzierung von 2-3 Knochenankern [8, 10,19,28].

In den letzten Jahren ist eine Vielzahl von biomechanischen Studien zu den Struktureigenschaften der Ankerfixation im Vergleich zum „Goldstandard“ der transossären Fixation durchgeführt worden $[10,21,28,33]$. Während Hart et al. [10] geringere Versagenskräfte nach Ankerfixation im Vergleich zur 3-fachen transossären Naht feststellen konnten und Lighthart et al. [21] vergleichbare Struktureigenschaften ermittelten, konnten Petri et al. in den durchgeführten Kadaverversuchen eine signifikant erhöhte Ausreißkraft der Titan- und Hydroxylapatitanker im Vergleich zu den transossären Nähten feststellen [28].

Zusammenfassend kann als Erkenntnis aus diesen Studien festgehalten werden, dass eine Ankerfixation mindestens vergleichbare Fixationskräfte bietet und als adäquate Alternative zu den als Goldstandard geltenden transossären Nähten gilt.

\section{Veraltete Ruptur/Reruptur}

Veraltete Rupturen oder Rerupturen stellen eine besondere Herausforderung für die Therapie dar. Wichtige Voraussetzung zur Behandlung einer Reruptur oder veralteten Quadrizepssehnenverletzung ist zum einen eine genaue Anamneseerstellung, um die genauen Ursachen der Reruptur, den Zeitpunkt oder die ausbleibende Heilung zu analysieren. Mögliche Ursachen können Nichteinhaltung des Rehabilitationsschemas, erneutes Trauma, Heilungsstörung aufgrund von Begleiterkrankungen oder Medikamenteneinnahme von heilungsinhibierenden Medikamenten (z.B. Kortison, Zytostatika, Immunsuppressiva) sein.

Nur durch eine sorgfältige Anamnese können Risikofaktoren für ein erneutes Versagen der Naht eingegrenzt und ggf. eliminiert werden.
Weiterhin muss eine aufwendige Analyse der Ruptur erfolgen. In den meisten Fällen ist eine MRT-Bildgebung der Quadrizepssehne zu empfehlen, um das weitere operative Vorgehen $\mathrm{zu}$ planen. Wichtige Aspekte sind dabei die Größe des Sehnendefekts, Ausmaß der Degeneration der verbliebenen Sehne und der Zustand des Reservestreckapparats.

Abhängig von dieser Analyse sollte danach das weitere operative Prozedere geplant werden. Falls sowohl das Ausmaß des Sehnendefekts als auch die Degeneration der Sehne geringgradig ausgeprägt sind, so kann in selteneren Fällen auch eine erneute primäre Adaptation der Sehne mit End-zu-End-Naht durchgeführt werden [16]. In den meisten Fällen ist eine direkte Naht jedoch nicht möglich, sodass der Sehnendefekt mit möglichst qualitativ hochwertigem Sehnenmaterial ausgefüllt werden sollte [30].

Im Falle einer gering ausgeprägten Degeneration des Sehnengewebes kann eine Überbrückung des Sehnendefekts mithilfe einer Z-Plastik oder einer gestielten Plastik mittels Separierung des profunden und oberflächlichen Anteils der Quadrizepssehne erreicht werden. Weiterhin kann ein Sehnendefekt auch mittels autologem Semitendinosussehnenoder Grazilissehneninterponat augmentiert werden [30] (Abb. 1). Eine primäre Readaptation der Sehne kann auch wahlweise durch einen V-förmigen Lappen aus dem proximalen Anteil der Sehne, welcher mit dem distalen Anteil und der patellaren Vorderfläche vernäht wird, verstärkt werden $[12,26,36]$.

\section{Nachbehandlung}

Die Nachbehandlung der Quadrizepssehnenruptur stellt einen Kompromiss zwischen Schonung der Nähte und evtl. Lappenplastiken und frühzeitigen Bewegungsübungen des Kniegelenks dar.

Insgesamt wird in der aktuellen Literatur der frühfunktionellen Beübung des Kniegelenks jedoch eine große Bedeutung beigemessen. Die frühzeitige passive Bewegungsübung der Sehne soll sowohl die Sehnenheilung induzieren als auch Adhäsionen verhindern [12,26,36]. Insbesondere die Einschränkung der Beweglichkeit des Kniegelenks führt im langzeitlichen Outcome zu einer erheblichen Verschlechterung der Kniefunktion und Zufriedenheit der Patienten. Aus diesem Grunde sollte bereits in der Früh-

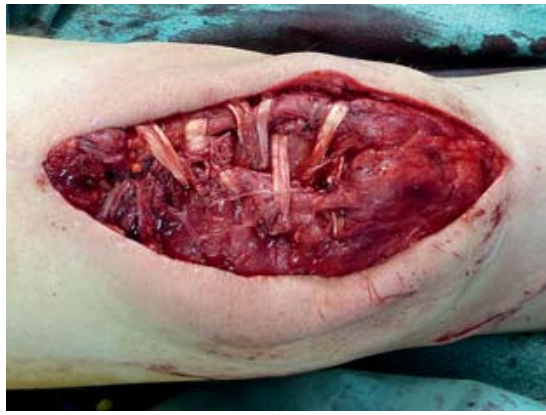

Abb. 1 Chronische Quadrizepssehnenruptur mit ausgeprägtem Substanzdefekt der Quadrizepssehne. Der Defekt wurde mittels biologischer Augmentationsplastik einer Semitendinosussehne verschlossen.

phase der postoperativen Behandlung eine intensive passive Bewegungsübung des Kniegelenks mittels CPM (Motorschiene) erfolgen. Diese Übungen können bereits in der Frühphase ergänzt werden mit isometrischen Anspannungsübungen des $\mathrm{M}$. quadriceps femoris.

Bezüglich der aktiven Belastung der betroffenen Extremität sollte je nach Befund (akuter Riss, chronischer Riss, Reruptur, Nebenerkrankungen etc.) eine Teilbelastung mit $20 \mathrm{~kg}$ an Unterarmgehstützen für 6-8 Wochen durchgeführt werden $[8,12,26]$. Um eine Überlastung der Sehne in den ersten postoperativen Wochen zu verhindern, sollte eine Orthese mit Limitierung der Flexion auf $60-90^{\circ}$ verwendet werden. Insbesondere bei Quadrizepssehnenrupturen kann kein allgemeingültiges Nachbehandlungsschema angegeben werden, sondern es sollte immer individuell auf den Patienten und seine Ruptur- und Nahtsituation angepasst werden. Schließlich haben Qualität des Sehnengewebes, Ausmaß der Degeneration und Rupturlokalisation wesentlichen Einfluss auf die Primärstabilität und den Heilverlauf.

Ein Monitoring der Sehnenheilung kann kostengünstig und suffizient mittels Sonografie erfolgen.

\section{Patellarsehnenruptur}

\section{Anatomie}

Die Patellarsehne stellt die Verlängerung der Quadrizepssehne dar, welche die Patella (Hypomochlion) mit der Tuberositas tibiae verbindet. Ihre Fasern stammen dabei aus der Endsehne des M. quadriceps femoris, wobei die meisten Fasern aus der flachen Endsehne des 
M. rectus femoris stammen. Die Patellarsehne hat eine zentrale Bedeutung bei der aktiven Kniestreckung, welche bei Ruptur der Sehne i.d.R. vollständig aufgehoben ist. Die Länge der Sehne steht i.d.R. in einem harmonischen Zusammenhang mit der Längenausdehnung der Patella. Dieses Verhältnis kann mittels Insall-Salvati- oder Caton-DechampsIndex berechnet werden $(1: 1)[5,13]$.

\section{Epidemiologie}

Die Patellarsehnenruptur ist eine eher seltene Verletzung des Kniegelenks. Ihr Anteil bez. der Verletzungen des Kniestreckapparats wird in der Literatur mit 3-6\% angegeben [11]. Jedoch sollte diese schwere Verletzung des Kniestreckapparats im Bewusstsein des behandelnden Arztes sein, da eine übersehene Patellarsehnen(teil)ruptur weitreichende Auswirkungen auf die Funktion des Kniegelenks haben kann. Erschreckenderweise wird die Quote der übersehenen Patellarsehnenrupturen mit bis zu 21\% angegeben. Im Gegensatz zu den Quadrizepssehnenrupturen, welche insbesondere im älteren Patientengut (über 40 Jahre) angetroffen werden, sind $80 \%$ der $\mathrm{Pa}$ tienten mit Patellarsehnenruptur unter 40 Jahre alt und gehören somit dem körperlich aktiveren Patientengut an [11]. Aus diesem Grund ist eine optimale Behandlung dieser Verletzung unabdingbar, um das angestrebte Aktivitätsniveau der Patienten aufrechtzuerhalten [11].

\section{Pathomechanismus}

Eine akute Patellarsehnenruptur kann sowohl durch direkte als auch indirekte Krafteinwirkung verursacht werden. Obwohl eine degenerative Schwächung der Sehne einen geringeren Einfluss im Vergleich zur Quadrizepssehne haben soll (80\% der Rupturen im Alter unter 40 Lebensjahren), so muss auch im Falle einer Patellarsehnenruptur von einer gewissen degenerativen Vorschädigung ausgegangen werden.

Die „anatomische Wetterecke“ der Patellarsehne scheint v.a. der Übergang vom unteren Patellapol zur Sehne zu sein. Hier werden die meisten Patellarsehnenrupturen im Erwachsenenalter angetroffen. Im Kindesalter treten Patellarsehnenrupturen häufig im distalen Bereich der Sehne am Übergang zur Tuberositas tibiae auf. In diesen Fällen handelt es sich i.d.R. um indirekte Krafteinwirkungen auf die Sehne. Intraligamentäre Durchtrennungen der Sehne kommen deutlich

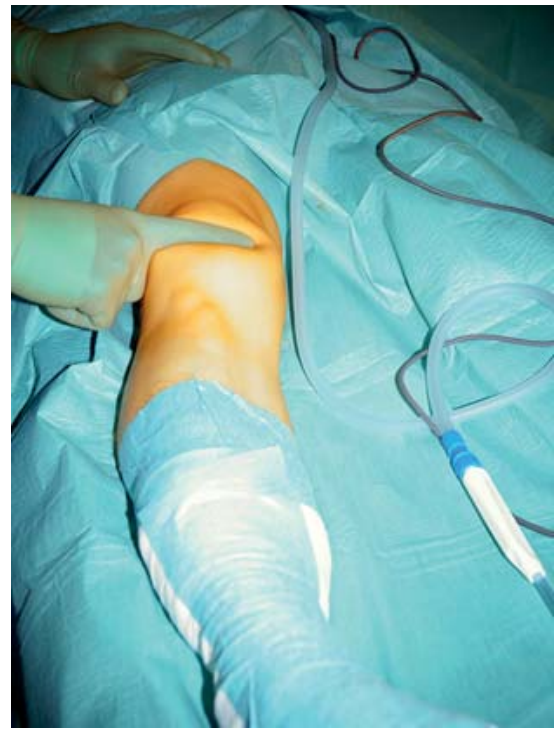

Abb. 2 Klinischer Befund einer Dellenbildung zwischen Patella und Tuberositas tibiae auf Grundlage einer akuten Patellarsehnenruptur

seltener vor und sind meistens Folge einer direkten (scharfen) Krafteinwirkung mit Zerschneidung der Sehne [18].

Wichtige Einflussfaktoren für eine degenerative Vorschädigung und damit Schwächung der Patellarsehne stellen internistische Nebenerkrankungen dar, welche in der Anamneseerhebung unbedingt beachtet werden müssen: $[6,17]$

- Diabetes mellitus

- Niereninsuffizienz

- rheumatoide Arthritis

- Ehlers-Danlos-Syndrom

- Lupus erythematodes

Weitere Ursachen von Schwächungen der Patellarsehnen können Patellarsehnenentnahmen $z$. B. bei VKB-Rekonstruktionen oder iatrogene Schädigungen im Rahmen einer Knieprothesenimplantation sein $[1,9]$.

\section{Diagnostik}

In Analogie zu der Quadrizepssehnenruptur spielt bei der Patellarsehnenruptur eine genaue Anamneseerhebung des Traumas, der Beschwerden, der Funktionseinschränkung und der gesamten Krankengeschichte eine große Rolle [11].

In den meisten Fällen kann eine vollständige Patellarsehnenruptur anhand einer dezidierten klinischen Untersuchung diagnostiziert werden. Folgende 3 Aspekte sind von besonderer Bedeutung und sollten stets erkannt werden:
- Einschränkung oder Unfähigkeit der aktiven Kniestreckung

- Patellahochstand (DD Quadrizepssehnenruptur)

- palpable Delle zwischen Patella und Tuberositas tibiae (Abb. 2)

- fehlender oder deutlich geringerer Erguss

In der Regel ist die aktive Streckung der betroffenen Extremität aufgrund der Verletzung vollständig aufgehoben.

Zur Erweiterung der klinischen Diagnostik ist eine projektionsradiologische Untersuchung des Kniegelenks in 2 Ebenen indiziert, um evtl. knöcherne Beteiligungen zu erkennen.

Zusätzlich kann mithilfe der seitlichen Röntgenaufnahmen die Höhe der Patella bestimmt werden. Hierzu sollte auch immer die Gegenseite im streng seitlichen Röntgenbild mit identischer Flexionsstellung des Kniegelenks in $30^{\circ}$ projektionsradiografisch als ,gesunder Vergleich" abgebildet werden. Mithilfe dieser Aufnahmen wird dann der InsallSalvati- oder Caton-Deschamps-Index bestimmt, um eine resultierende Patella alta erkennen zu können $[5,13]$.

Zur genaueren Analyse von intratendinösen Rupturen empfiehlt sich die Durchführung einer sonografischen Untersuchung der Sehne. Nur im zweifelhaften akuten Fall (z.B. Teilruptur) oder im Fall einer chronischen Ruptur oder Reruptur ist die Durchführung einer MRT-Untersuchung streng indiziert. Mit ihrer Hilfe kann die Dehiszenzstrecke und v.a. auch der Degenerationsstatus der Sehne genau analysiert werden. Diese Informationen sind jedoch für die Planung des operativen Vorgehens von entscheidender Bedeutung.

\section{Therapie}

Grundsätzlich besteht im Falle einer vollständigen Ruptur der Patellarsehne eine Operationsindikation. Kontraindikationen bestehen lediglich im Falle von schweren Begleitverletzungen oder Begleiterkrankungen, welche den Patienten vital gefährden. Jedoch sollte auch in diesen Fällen nach Stabilisierung des Patienten oder Konsolidierung der Weichteilverhältnisse eine verzögerte operative Versorgung angestrebt werden, um einen Funktionserhalt der Extremität zu gewährleisten. 


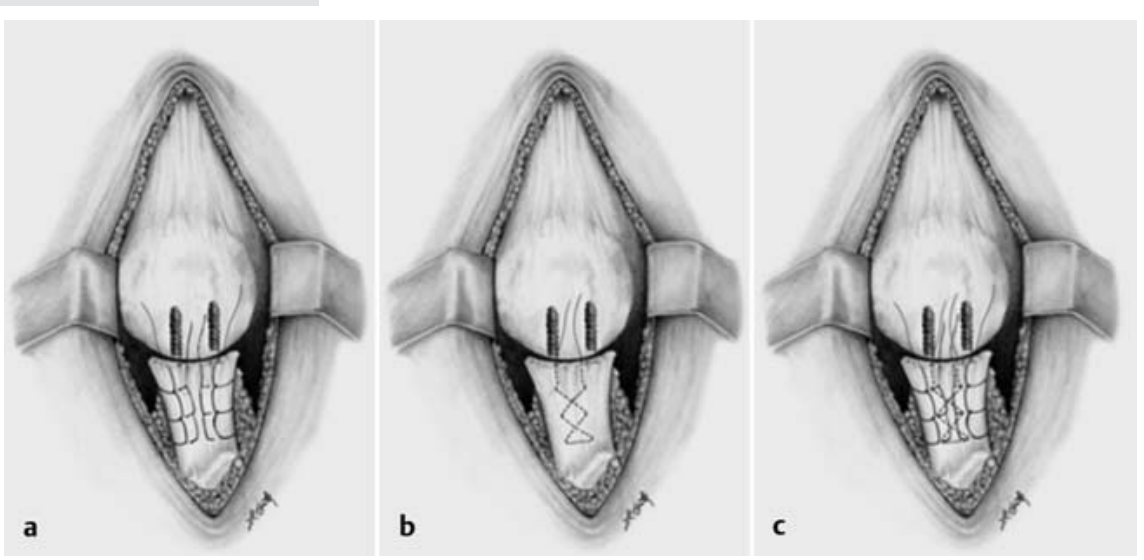

Abb. 3 a bis c Schematische Zeichnung proximaler Patellarsehnenrupturen mit Knochenankerfixation und Bunnel- und Krackow-Nähten aus Gaines et al. [7]: a Durchflechtung der Patellarsehnenränder und der zentralen Anteile mit Krackow-Nähten. b Bunnel-Naht zur Armierung des zentralen Sehnenanteils. c Ergebnis kurz vor dem Festknoten mit vorgelegten Bunnel- und Krackow-Nähten.

\section{Akute Ruptur}

Die Versorgung der akuten Patellarsehnenruptur folgt seit 1950 einem vergleichbaren Schema. Grundsätzlich sollte eine Adaptation der Sehnenenden erreicht werden. Handelt es sich um eine intraligamentäre Rupturform, erfolgt eine Naht der Sehne mittels U-Nähten, Bunnell- oder Kessler-Nähten. Die Nähte können sowohl mit resorbierbarem (z.B. PDS) oder auch nicht resorbierbarem Nahtmaterial (z.B. Ethibond) durchgeführt werden.

Im Falle einer knochennahen Ruptur (Avulsionsverletzung) unmittelbar im Bereich der tibialen oder patellaren Insertion sollte eine knöcherne Fixation der Nähte erfolgen. Hierbei ist sowohl die transossäre Naht als auch die Verwendung von Knochenankern möglich (Abb. 3) [3, 4,7]. Biomechanische Versuche haben dabei vergleichbare biomechanische Eigenschaften feststellen können [3].

Aufgrund der eingeschränkten Primärstabilität der Nähte wird eine zusätzliche Stabilisierung der Naht mittels McLaughlin-Cerclage empfohlen [24].

In der Literatur finden sich unterschiedliche Konfigurationen dieser überbrückenden Cerclage. So kann diese als Rahmennaht sowohl im Bereich des unteren Patelladrittels als auch in der Tuberositas durch eine transossäre Bohrung geführt werden. Alternativ kann auch eine Achtertour vom Quadrizepssehnenansatz (transtendinös, ohne transossäre Bohrung) zur Tuberositas tibiae geführt werden. Zur Erhöhung der biomechanischen
Stabilität kann die Cerclage im Bereich der Tuberositas durch eine kanülierte Kleinfragmentschraube geleitet werden [29].

Neben der ursprünglichen Verwendung einer Draht-Cerclage, welche einer Implantatentfernung im Rahmen eines operativen Eingriffs bedarf, kann alternativ auch eine PDS-Cerclage mit einer kräftigen PDS-Kordel (z.B. 0,7 oder $1,5 \mathrm{~mm}$ ) durchgeführt werden, wodurch eine Folgeoperation nicht notwendig ist. Allerdings erkauft man sich diesen Vorteil mit einem erhöhten Risiko für eine Fremdkörperreaktion durch das elastischere PDS-Material [11].

Neben der vollständigen Adaptation der Sehne stellt die genaue Einstellung der Patellahöhe im Seitenvergleich einen wichtigen Schritt der Patellarsehnenrekonstruktion dar. Insbesondere bei stark aufgefaserten Sehnenenden ist eine korrekte Höheneinstellung der Patella schwierig (Abb.4). Bei der Einstellung der Patellahöhe sollte in jedem Fall eine intraoperative fluoroskopische Kontrolle erfolgen.

Zur vergleichenden Kontrolle der physiologischen Patellahöhe sollten die intraoperativen Aufnahmen entweder mit präoperativen Aufnahmen der Gegenseite in identischer Beugestellung verglichen werden oder alternativ das kontralaterale Kniegelenk frei beweglich zum intraoperativen Vergleich abgewaschen werden.

Eine inkorrekte Einstellung der Patella kann im weiteren postoperativen Verlauf zu schweren Komplikationen für den

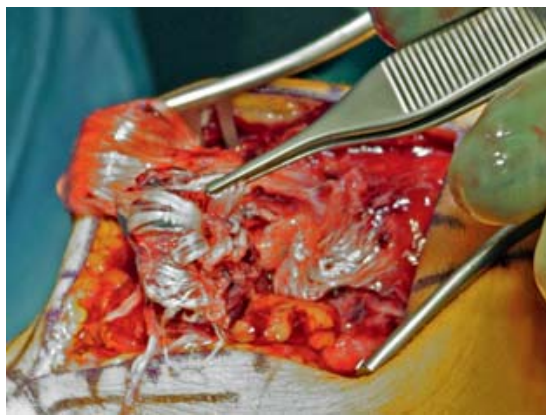

Abb.4 Intraoperativer Befund einer akuten Patellarsehnenruptur mit typisch aufgefaserten Sehnenenden.

Patienten führen („Overstuffing“; Retropatellararthrose, Patellainstabilität mit Luxationsneigung etc.)

\section{Chronische Ruptur/Reruptur}

Chronische Rupturen (verzögert versorgte Rupturen) oder Rerupturen sind besonders schwierig $\mathrm{zu}$ behandeln. Wichtige Einflussfaktoren für das operative Vorgehen sind hierbei das Ausmaß der Dehiszenz der Sehnenstümpfe, die allgemeine Qualität des Sehnengewebes, insbesondere im Rupturbereich, und die weiteren Begleitverletzungen. Zur genauen Analyse dieser Einflussfaktoren sollte im Falle einer chronischen oder veralteten Ruptur eine MRT-Diagnostik durchgeführt werden.

Im Falle einer weiten Dehiszenzstrecke zwischen den Sehnenstümpfen und/ oder einer hochgradig degenerativen Veränderung der Sehnenstümpfe ist eine primäre Adaptation der Sehnen nicht möglich (Abb.5). Somit sollte eine Augmentation der Sehne zur Überbrückung des dehiszenten Sehnenbereichs mit autogenem Sehnenmaterial erfolgen. Hierzu eignen sich v.a. die Sehnen des M. gracilis oder M. semitendinosus. Diese Sehnen können entweder geschwenkt oder nach Absetzen vom Ansatz transossär fixiert werden (Abb. 6 und 7). Auch diese Augmentationstechniken sollten zur Erhöhung der biomechanischen Stabilität mit einer zusätzlichen PDS- oder Draht-Cerclage abgesichert werden (Abb. 8).

\section{Nachbehandlung}

Die Nachbehandlung einer Patellarsehnenruptur ist eine „Gratwanderung“ bestehend aus der Notwendigkeit der Schonung der eingeschränkt stabilen Sehnennähte und der Notwendigkeit der Mobilisation des Gelenks auf der anderen Seite. 


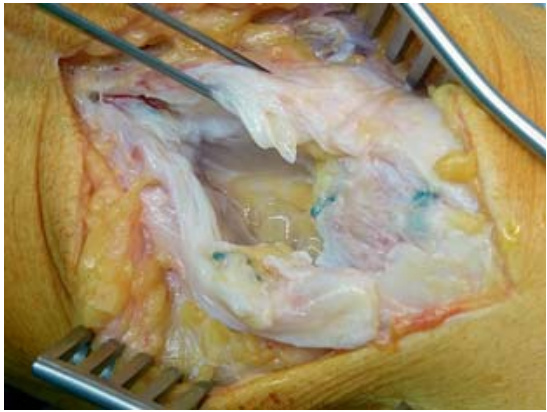

Abb. 5 Chronische Patellarsehnenruptur mit Dehiszenz und degenerativ verändertem Sehnengewebe: Eine primäre Naht ist so nicht möglich.

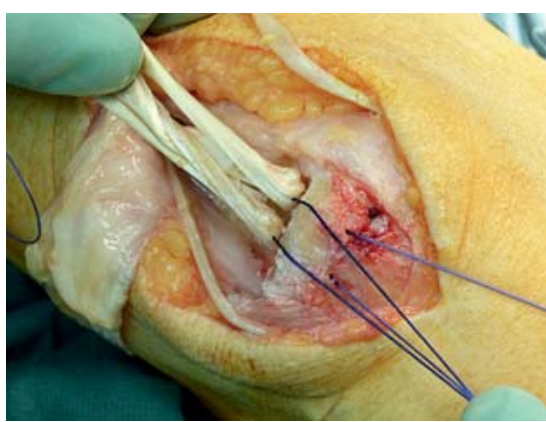

Abb. 7 Einnähen des autologen Sehnengewebes nach Umschlagen der Semitendinosussehne zur Augmentationsplastik der Patellarsehne.

Bei zu passiver Nachbehandlung erhöht sich die Gefahr für postoperative Komplikationen signifikant.

Hierzu gehören: [11]

- Hämatome (6\%)

- oberflächliche Infektionen (5\%)

- Thrombose/Embolien (5-29\%)

- Quadrizepsatrophien (14-56\%)

- Flexionsdefizit (21-33\%)

- Extensionsdefizit (4\%)

Somit sollte eine differenzierte aggressive Nachbehandlung erfolgen. Im eigenen Vorgehen werden die Patellarsehnenrupturen adaptiert an den intraoperativen Befund für 1 Woche in der MecronSchiene und anschließend für jeweils 2 Wochen mit einer Hartschalenrahmenorthese mit 30, 60 und $90^{\circ}$ Flexionseinschränkung mobilisiert. Für 6 Wochen sollte in den Schienen eine Teilbelastung mit $20 \mathrm{~kg}$ durchgeführt werden. Je nach Befund kann diese Teilbelastung auch schon ab der 4 . Woche gesteigert werden.

Ein weiterer wichtiger Bestandteil der frühen postoperativen Behandlung ist die passive Beübung der Flexion mittels CPM- oder Camoped-Schiene (Oped,

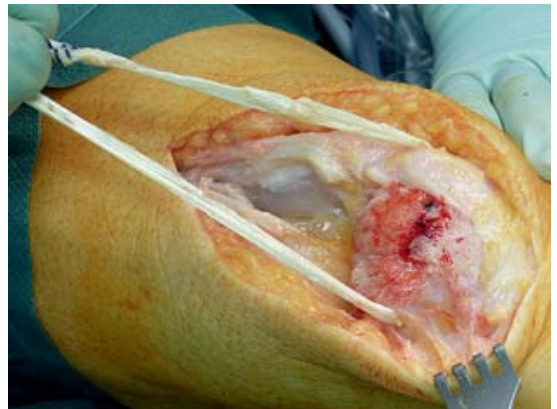

Abb. 6 Transossäre Fixation einer Semitendinosussehne zur Augmentation einer chronischen Patellarsehnenruptur mit Dehiszenz der Sehnenstümpfe.

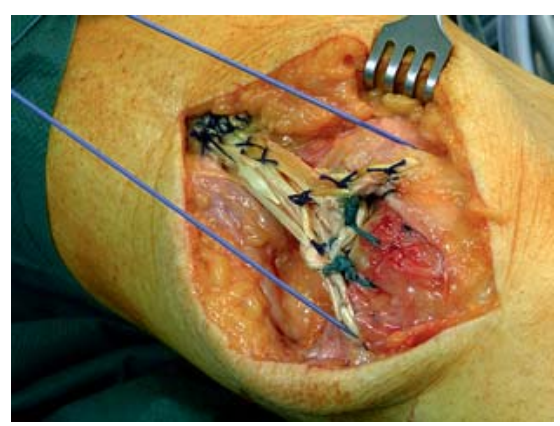

Abb. 8 Nach dem Einnähen des autologen Sehnengewebes wird die Augmentationsplastik mittels einer PDS-Cerclage $(1,5 \mathrm{~mm}$ PDSKordel) im Sinne einer McLaughlin-Cerclage verstärkt.

Valley). Hiermit kann ohne übermäßige Belastung der Rupturstelle die Beweglichkeit des Kniegelenks gefördert und somit Einschränkungen der ROM erfolgreich verhindert werden.

\section{Verletzung des Kniestreckapparats} bei liegender Knieprothese

Verletzungen des Kniestreckapparats sind schwerwiegende Komplikationen der Knieendoprothetik, welche glücklicherweise in der aktuellen Literatur mit einer geringen Inzidenz von 0,17$2,5 \%$ angegeben werden $[2,23]$.

Die klinischen Ergebnisse wie auch der Heilverlauf sind jedoch nicht mit einer Verletzung des Kniestreckapparats ohne prothetische Versorgung zu vergleichen. Es ist hierbei mit deutlich schlechteren Ergebnissen zu rechnen mit signifikanten Einschränkungen der Funktion des Kniegelenks. Eine Ruptur der Patellarsehne kommt deutlich häufiger vor als eine Ruptur der Quadrizepssehne [1]. Grundsätzlich werden im Falle einer Sehnenruptur bei liegender Knieprothese vergleichbare Operationstechniken angewendet.
Die konservative Therapie kann im Falle einer Teilruptur mit einem Streckdefizit kleiner $20^{\circ}$ durchgeführt werden.

Komplette Rupturen der Quadrizepsoder Patellarsehne sollten einer operativen Intervention zugeführt werden. Hierbei sind, analog zu den nicht periprothetischen Rupturen, neben direkten Nahttechniken in vielen Fällen autologe Sehnenaugmentationsplastiken, Muskellappenaugmentationen oder auch die Implantation von synthetischen Ersatzmaterialien indiziert [25].

\section{Literatur}

${ }^{1}$ Bieger R, Kappe T, Wernerus $D$ et al. [Treatment of extensor mechanism rupture after total knee arthroplasty]. Z Orthop Unfall 2013; 151: 475-479

2 Brooks P. Extensor mechanism ruptures. Orthopedics 2009; 32: 683-684

${ }^{3}$ Bushnell BD, Byram IR, Weinhold PS et al. The use of suture anchors in repair of the ruptured patellar tendon: a biomechanical study. Am J Sports Med 2006; 34: 1492-1499

${ }^{4}$ Bushnell BD, Tennant JN, Rubright JH et al. Repair of patellar tendon rupture using suture anchors. J Knee Surg 2008: 21: 122-129

${ }^{5}$ Caton J, Deschamps G, Chambat $P$ et al. Les rotules basses: a propos de 128 observations. Rev Chir Orthop Reparatrice Appar Mot 1982; 68: 317-325

${ }^{6}$ Cree C, Pillai A, Jones B et al. Bilateral patellar tendon ruptures: a missed diagnosis: case report and literature review. Knee Surg Sports Traumatol Arthrosc 2007; 15: 1350-1354

${ }^{7}$ Gaines RJ, Grabill SE, DeMaio M et al. Patellar tendon repair with suture anchors using a combined suture technique of a KrackowBunnell weave. J Orthop Trauma 2009; 23: 68-71

8 Grim C, Lorbach O, Engelhardt M. [Quadriceps and patellar tendon ruptures]. Orthopade 2010; 39: 1127-1134

${ }^{9}$ Haasters F, Ockert B, Mutschler W et al. Patellarsehnenruptur 10 Jahre nach vorderem Kreuzbandersatz durch Patellarsehnen-Knochen-Transplantat. Unfallchirurg 2009; 112: 728-733

${ }^{10}$ Hart ND, Wallace MK, Scovell JF et al. Quadriceps tendon rupture: a biomechanical comparison of transosseous equivalent doublerow suture anchor versus transosseous tunnel repair. J Knee Surg 2012; 25: 335-339

11 Heinrichs G, Kiene J, Hillbricht S et al. Patellarsehnenruptur. Trauma Berufskrankh 2010; 12 (Suppl. 4): S453-S456

12 Herbort M, Raschke MJ. [Ligament ruptures of the lower extremity in the elderly]. Unfallchirurg 2011; 114: 671-680

${ }^{13}$ Insall J, Salvati E. Patella position in the normal knee joint. Radiology 1971; 101: 101104

${ }^{14}$ Józsa L, Kannus P. Histopathological findings in spontaneous tendon ruptures. Scand J Med Sci Sports 1997; 7: 113-118

${ }^{15}$ Kannus P, Józsa L. Histopathological changes preceding spontaneous rupture of a tendon. A controlled study of 891 patients. J Bone Joint Surg Am 1991; 73: 1507-1525

16 Kerin C, Hopgood P, Banks AJ. Delayed repair of the quadriceps using the Mitek anchor system: a case report and review of the literature. Knee 2006; 13: 161-163 
17 Khan KM, Maffulli N, Coleman BD et al. Patellar tendinopathy: some aspects of basic science and clinical management. Br J Sports Med 1998; 32: 346-355

18 Kiene DJ, Paech A, Wenzl ME. Patellarsehnenruptur. Trauma Berufskrankh 2005; 7: 162 167

${ }^{19} \mathrm{Kim}$ TW, Kamath AF, Israelite CL. Suture anchor repair of quadriceps tendon rupture after total knee arthroplasty. J Arthroplasty 2011; 26: 817-820

20 Konrath GA, Chen D, Lock $T$ et al. Outcomes following repair of quadriceps tendon ruptures. J Orthop Trauma 1998; 12: 273-279

21 Lighthart WA, Cohen DA, Levine RG et al. Suture anchor versus suture through tunnel fixation for quadriceps tendon rupture: a biomechanical study. Orthopedics 2008; 31: 441

22 Lowry AW, McFarland EG, Cosgarea AJ et al. Partial rupture of the quadriceps tendon in a tennis player. Clin J Sport Med 2001; 11: 277-279

23 Malhotra R, Garg B, Logani V et al. Management of extensor mechanism deficit as a consequence of patellar tendon loss in total knee arthroplasty: a new surgical technique. J Arthroplasty 2008; 23: 1146-1151

24 McLaughlin HL. Repair of major tendon ruptures by buried removable suture. Am J Surg 1947; 74: 758-764

${ }^{25}$ Nam D, Abdel MP, Cross MB et al. The management of extensor mechanism complications in total knee arthroplasty. AAOS exhibit selection. J Bone Joint Surg Am 2014; 96: e47
${ }^{26}$ Ochman DS, Langer M, Petersen W et al. Quadrizepssehnenruptur. Unfallchirurg 2005; 108: 436-444

27 Petersen $W$, Stein V, Tillmann B. [Blood supply of the quadriceps tendon]. Unfallchirurg 1999; 102: 543-547

28 Petri M, Dratzidis A, Brand S et al. Suture anchor repair yields better biomechanical properties than transosseous sutures in ruptured quadriceps tendons. Knee Surg Sports Traumatol Arthrosc 2015; 23: 1039-1045

${ }^{29}$ Ramseier LE, Werner CM, Heinzelmann $M$. Quadriceps and patellar tendon rupture. Injury 2006; 37: 516-519

30 Rehman H, Kovacs P. Quadriceps tendon repair using hamstring, prolene mesh and autologous conditioned plasma augmentation. A novel technique for repair of chronic quadriceps tendon rupture. Knee 2015 [Epub ahead of print]

${ }^{31}$ Reider B, Marshall JL, Koslin B et al. The anterior aspect of the knee joint. J Bone Joint Surg Am 1981; 63: 351-356

32 Rougraff BT, Reeck CC, Essenmacher J. Complete quadriceps tendon ruptures. Orthopedics 1996; 19: 509-514

33 Sellei RM, Bauer E, Hofman $M$ et al. Reconstruction of a quadriceps tendon tear using Polyvinylidene fluoride sutures and patellar screw fixation: a biomechanical study. Knee 2015 [Epub ahead of print]

34 Swamy GN, Nanjayan SK, Yallappa $S$ et al. Is ultrasound diagnosis reliable in acute extensor tendon injuries of the knee? Acta Orthop Belg 2012; 78: 764-770
${ }^{35}$ Xu Y, Murrell GA. The basic science of tendinopathy. Clin Orthop Relat Res 2008; 466: 1528-1538

36 Yilmaz C, Binnet MS, Narman S. Tendon lengthening repair and early mobilization in treatment of neglected bilateral simultaneous traumatic rupture of the quadriceps tendon. Knee Surg Sports Traumatol Arthrosc 2001; 9: 163-166

Priv.-Doz. Dr. med. Mirco Herbort Funktionsoberarzt

Dr. med. Christoph Domnick Assistenzarzt

Univ.-Prof. Dr. med.

Michael J. Raschke

Ärztlicher Direktor

Klinik für Unfall-, Hand- und

Wiederherstellungschirurgie

Universitätsklinikum Münster

Waldeyerstraße 1

48149 Münster

Mirco.Herbort@uni-muenster.de 